\title{
Versuche der Teilhabe in technologischen Zeitaltern
}

Wie finden wir Antworten auf technologische Prozesse, die gemeinhin mit

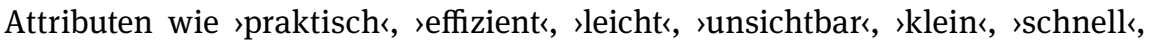

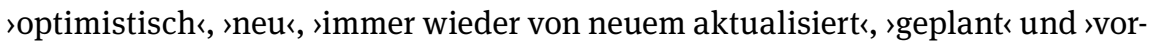
herbestimmt versehen werden? Gibt es nicht auch eine Suche nach Antworten darauf, die sich mit gegenteiligen Attributen wie ınutzlos`, >ineffizient‘, ১schwer`,

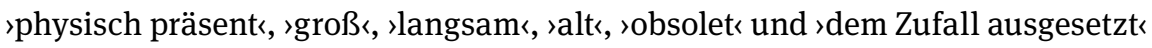
beschreiben lässt? Was motiviert eine solche Suche, die nicht nur gegenteilig, sondern vielleicht auch santiquiert erscheinen mag? Diesen Fragen geht mein Beitrag in zwei Schritten nach und zwar, erstens, indem diese vermeintlich santiquierte`Suche beispielhaft vollzogen wird, im Laufe derer sich eine Teilhabe an einem auch sunproduktiven Verhalten herausstellt, die sich gegen technologische Ideen, die unsere Gegenwart anscheinend definieren, richtet, und die, zweitens, diese Ideen damit in neue Richtungen lenken, die sich mit Vorstellungen von Freiheit und der (Heraus-)Bildung des Selbst in Beziehung zu Anderen in bewusst ausgewählten Gemeinschaften verbindet und dabei andere Orte schafft, die Technologien in einem nicht produktiven, nicht gewinnorientierten Sinn nutzen. ${ }^{1}$ Über die Suche nach diesen anderen Orten, angefangen mit Jessica Sequeira und ihren poetischen Ansätzen zu einem Denken in technologischen Zeitaltern bis zu den Versuchen François Julliens darüber, neue Wege im Denken des Gemeinsamen einzuschlagen, werden auf diese Weise Möglichkeiten alternativer Orte herausgearbeitet, wo sich kreative Antworten finden lassen, und zwar nicht, um letztlich jegliche Technologien abzulehnen, sondern um den Blick auf sie zu schärfen und sie anders beziehungsweise anderswo anzuwenden. Unter dem Begriff >Ort` fasse ich Antworten auf die Frage, wo sich etwas oder jemand befindet und etwas stattfindet. Unter dem Begriff der >Technologie` versammle ich Vorstellungen, Verfahren und Vorrichtungen, mit denen sich Möglichkeiten, Grenzen und Verbindungen der Technik ausloten lassen, wobei ich den Begriff `Technikı von der antiken griechischen Konzeption der techne als Darstellung und auch kunstvolle Herstellung und Gestaltung herleite. Aus den kommenden Beispielen werden drei ausgewählte medienwissenschaftliche

1 Der Beitrag basiert auf meinem gleichnamigen Habilitationsvortrag, gehalten im Fachbereich Literaturwissenschaft an der Universität Konstanz am 18.12.2019.

https://doi.org/10.1515/jbmp-2020-0017 
Forschungs- und Lehrperspektiven entwickelt, die an der Schnittstelle zu den Dis/Ability Studies stehen, Perspektiven auf Zugänglichkeit, Teilhabe und Wirksamkeit.

\section{I.}

Jessica Sequeira, Autorin und Übersetzerin, veröffentlicht 2018 einen Band unter dem Titel Other Paradises. Poetic Approaches to Thinking in a Technological Age, der die eingangs gestellten Fragen aufwirft und nach entsprechenden Antworten sucht. ${ }^{2}$ Ihr Vorgehen ist dabei ein poetisches und essayistisches, das über seine intendierte kompositorische Strenge exemplarische Situationsbeschreibungen miteinander verflechtet und verdichtet, statt sie ausschließlich definitorisch und methodisch abzuleiten. Sequeira bringt auf diese Weise über Schnitt und Montage Korrespondenzen für uns als Leserinnen und Leser aufs Papier, die es durch ihre offene Gestaltung uns überlässt, wie wir damit umgehen. Meine Herangehensweise im Essay, mein versuchtes genaues Lesen, Herauslesen und Aufschreiben, nimmt diesen Vorschlag der Autorin auf, der sich auch dadurch auszeichnet, dass sie die Anderen Paradiese, von denen im Haupttitel des Bandes die Rede ist, in Ländern wie Japan, Mexiko, Indien, Frankreich, Neuseeland oder der Türkei aufsucht, und zwar ausgehend von dem Paradies, an dem sie aufgewachsen ist, im Silicon Valley, dem Technologiezentrum in der kalifornischen Bay Area um San José und San Francisco, Standort von IT und Hightech, Sitz für Unternehmen wie Adobe, Apple, Google, Ebay, Facebook, Hewlett-Packard, Tesla, Yahoo und vielen anderen mehr.

Nun ist dieses Paradies keines, in dem Sequeira verweilen möchte, denn es dreht sich dort in den technologischen Prozessen, die sich unter anderem mit der Herstellung und Vermarktung von zum Beispiel Software, Smartphones, 3-D-Druckern und Elektroautos befassen, alles um die anfangs schon aufge-

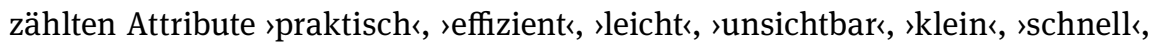

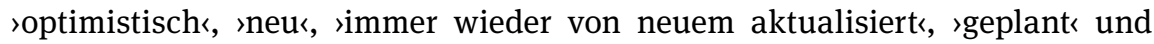
ıvorherbestimmt . Der kanadische Autor Douglas Coupland hat vor allem in den 1990er Jahren mit genau diesen Attributen gearbeitet, indem er zum Beispiel 1995 in seinem Roman Microserfs (in deutscher Übersetzung: Mikrosklaven) von einer Gruppe von Programmierern bei Microsoft erzählt, die ein Leben jenseits des PCs suchen und sich daher von Seattle ins Silicon Valley aufmachen, um

2 Vgl. meine Übersetzung ihrer Einleitung und eines Kapitels daraus in diesem Band. 
dort an einer Multimediasoftware zu arbeiten, die in ihrem virtuellen Aufbau Lego-Bausteinen ähnelt. »Arm oder nicht, das Leben ist wieder der totale Programmierwahnsinn - nur daß wir uns diesmal für uns selbst abrackern anstatt für irgendeinen Riesenkonzern, für den wir ebensogut austauschbare, blutleere PlaySkool-Figuren sein können. Wir haben, einen Tag nachdem wir hier angekommen sind, mit dem Programmieren angefangen. ${ }^{3}$ Bei beiden geht es um die Perspektive von vor allem 20- bis 30-jährigen; altersbedingt gehören bei Coupland die PCs, CD-ROMs und Klapphandys, bei Sequeira eher die ipads, Cloud-Dienste und Smartphones zur stechnologischen Sozialisation< in verschiedenen `Zeitaltern`, das heißt überblickshalber eingrenzbaren Zeiträumen, wie hier den 1980er, 1990er und 2000er Jahren. Sequeira eröffnet ihre Gedanken dazu folgendermaßen: „Zum Aufwachsen in Kalifornien gehörte der Glaube an Technologie zur Landschaft, genauso wie die Mammutbäume oder Swimming Pools. Dieser unhinterfragte Glaube existierte in den Unternehmensgebäuden, die die Straßen säumten, in den Internet Cafés, in den Bildern von David Hockney [...]. Ob jemand sich aussucht, neue Gadgets willkommen zu heißen oder nicht, ob jemand in Silicon Valley wohnt oder nicht, Technologie und die Antworten der Menschen auf sie haben einen Effekt. « ${ }^{4}$

Beispielhaft sucht Sequeira in der Folge in einem Kapitel Istanbul auf, in der sie über Menschen schreibt, die sich der sogenannten Maker- oder Do-It-Yourself-Bewegung zurechnen, die versuchen, »existierende Technologie für persönlichen und nicht-kommerziellen Gebrauch zu nutzen, entweder aus Neugier oder um spezifische Probleme wie Krankheiten zu adressieren. Ihre Arbeit weckt die Aufmerksamkeit dafür, wie man eine Vielzahl an kreativen und gemeinschaftlichen Einflüssen willkommen heißen kann und zugleich, wie man bemerken kann, was fehlt, um zu kreieren, was eine Person oder Gesellschaft brauchen könnte. ${ }^{5}$

Sequeira berichtet über Zeynep Karagöz, die in Istanbul eine Wohltätigkeitsorganisation namens Robotel Türkiye leitet und versucht, Kinder mit Behinderungen an den Fingern oder an der Hand durch mit 3-D-Druckern hergestellten Prothesen zu helfen, Prothesen, die in ersten Schritten von den Makern initiiert durch 3-D-Software am Computer digital, das heißt mathematisierbar, berechnet und gestaltet werden und in weiteren Schritten durch etwas Anderes, nämlich Kunststoffe, Metalle oder Keramik analog, das heißt materiell, in einem mehrstufigen Prozess im 3-D-Drucker geformt werden, um schließlich als Prothesen

3 Douglas Coupland, Mikrosklaven, München 1999, S. 171.

$4 \mathrm{Vgl}$. Sequeira in diesem Band.

5 Vgl. ebd. 
am Körper der Kinder angepasst zu werden. Es geraten so die medialen und performativen Situationen und ihre konkreten Settings selbst in den Blick, im Laufe derer nicht mehr nur gefragt wird, was erscheint und erfahren wird, sondern wie und wodurch etwas als etwas erscheint und erfahren wird.

Sequeiras Vorgehensweise ist hingegen eine, die sich von der Korrespondenz mit Karagöz inspirieren lässt, aber dokumentarisches Material wie Zeitungsartikel oder Videos hinzunimmt, um mit ihrer Hilfe weiterzudenken und fortzuschreiben, so auch mit einem Ausschnitt eines auf YouTube einsehbaren Videos der Maker-Gemeinschaft e-NABLE, der zwischen Ausführungen eines Jungen namens Kieran und den an der Maker-Gemeinschaft e-NABLE beteiligten Personen changiert. ${ }^{6}$ e-NABLE, deren Name das Befähigen und Ermöglichen durch elektronische Mittel in sich trägt, ist eine immer noch bestehende globale online Gemeinschaft zur Herstellung von Prothesen, von der auch das Video (2015) stammt. Finanziert über Spenden, arbeitet sie mit Designern des Auto Desk Technology Center zusammen, mit Vertreter:innen der Industrie, Wissenschaft und Wirtschaft mit Sitzen in San Francisco, Boston, Toronto und Birmingham, mit denen es in Workshops um den Entwurf von Prothesen geht, die diese kostengünstig herstellen und kostenlos zugänglich machen. Das Video beginnt mit Kieran, der erzählt, dass er, bevor er eine Handprothese dieser Machart bekam, bevor er überhaupt eine Hand besaß, »es schwer hatte«, am Alltagsleben teilzunehmen.

Kieran wurde mit dem Amniotischen Band Syndrom geboren und hat daher an der rechten Hand keine Finger. Er erzählt uns, dass er deswegen oft gehänselt werde, dass er gerne an einer Gemeinschaft teilhaben möchte, gerne Freunde, eine rechte Hand, überhaupt eine Hand hätte, um die er sich nicht zu kümmern brauche. Im Video wird berichtet, dass moderne Prothesen zehntausende Dollars kosten und Kinder schnell aus ihnen herauswachsen. Für die meisten Kinder ist es daher ein unmöglicher Traum, an die Prothesen heranzukommen. Es folgt ein Gespräch mit dem Gründer von e-NABLE, der die Arbeit der Freiwilligen beschreibt, die 3-D-Drucker und 3-D-Design Software nutzen, Prothesen für Kinder und Erwachsene herzustellen, um sie an die Betroffenen kostenlos weiterzugeben. Die Prothesen seien kostengünstig zu produzieren, unter einhundert Dollar, da sie rein körpergesteuert, mechanisch sind und keine Motoren oder Batterien benötigen. Es sind leichte, einfache Hilfsmittel, die nicht einmal vorgeben, wie normale Hände auszusehen. Sie sehen ganz anders aus, etwa wie Dinge, die sIronman oder andere Superhelden tragen. Die Kinder mögen sie genau aus diesem Grund. Kieran erzählt, dass er, sobald er diese `Hand hatte,

6 Vgl. https://www.youtube.com/watch?v=Cl8ijPGEKO8 (letzter Zugriff: 10.04.2020). 
Freunde ihn fragten, wie es ihm ginge und ob sich etwas verändert habe, wie er mit der Prothese zurechtkäme. »Ich liebe sie. Ich kann Dinge damit greifen, ich kann helfen.« Wir sehen ihn in der Küche, wie er sich mit einem Mixer ein Getränk zubereitet. Kieran besucht mit seiner Familie einen Workshop, um Prototypen auszutesten, ihre Wirksamkeiten zu erproben, um »die Perspektive aufs große Ganze zu erlangen «. - Technology in Our Hands. Die Technologie in unseren Händen, was zeige, so die Hersteller, dass ıgewöhnliche`Menschen, Laien, >aufregende` Dinge entwerfen können, wenn sie nur Zugang zu den entsprechenden Werkzeugen bekommen. Make something to make a difference. Mache etwas, um einen Unterschied zu machen.

Hieraus ergeben sich die eingangs erwähnten Perspektiven auf Zugänglichkeit, Teilhabe und Wirksamkeit:

Zunächst eröffnet sich die Perspektive über die Zugänglichkeit zu Technologien, wie sie in der Maker-Bewegung thematisiert und ermöglicht wird. Wenn wir, ausgehend von sozialer Ungleichheit und einem Ungleichgewicht in der Verteilung von Arbeit und Kapital, eine durchgehende Computerisierung unserer Lebenswelt feststellen, in deren Zentrum die Digitalität steht, die eine Herrschaft der Codes, der Programme und der Algorithmen und damit auch der Kontrolle und Überwachung mit sich bringt, so zeigen die angeführten Beispiele doch Möglichkeiten auf, wie diese Codes, Programme und Algorithmen anders verwendet werden können. Es gilt daher, weiter zu suchen und auszuprobieren, wo diese Zugänglichkeit möglich ist und ermöglicht werden kann, was auch damit einher geht, die sowohl »epistemische als auch handlungspraktische Eingebundenheit technischer Medien zu überprüfen« und damit »das Verhältnis von divers fähigen Körpern, Technik und Sinneserfahrungen auszuloten«, einer Arbeit, der sich beispielsweise die Arbeitsgruppe »Medienwissenschaft und Dis/ Ability Studies « mit ihren Sprechern Jan Müggenburg und Robert Stock in der Gesellschaft für Medienwissenschaft verschrieben hat. ${ }^{7}$

Andere Orte in diesem Kontext aufzusuchen, heißt dann auch, nach Heterotopien Ausschau zu halten, die sich, nach Michel Foucault, von Utopien dadurch unterscheiden, dass sie real existierende Orte sind und damit nicht einfach Produkte der Imagination. Diese real existierenden Orte geben Heterogenitäten Raum, für die in einem gegebenen Raumgefüge kein Platz vorgesehen ist: entweder so, wie Tobias Nikolaus Klass verdeutlicht, »dass dem abweichenden Raum für autonome Entfaltung gegeben wird oder aber so, dass genau im Gegenteil alles Heterogene an einem Ort versammelt wird, um es dort wieder ins Herrschende

7 Vgl. die Website der Arbeitsgruppe: http://gfmedienwissenschaft.de/ag-medienwissenschaftund-disability-studies (letzter Zugriff: 10.04.2020). 
einzugliedern. $\ll^{8}$ Die erste Form nennt Foucault 〉Krisenheterotopie`, die zweite >Abweichungsheterotopieく. Vor allem die zweite Form dient mit dem von Jeremy Bentham in den 1790er Jahren entworfenen Panoptikum als räumliche Ausfaltung der Macht, die sich über Disziplinierungsräume etabliert, denen die >Krisenheterotopie، als Ort des Widerstands gegenübersteht. ${ }^{9}$

Andere Orte in diesem Kontext aufzusuchen, heißt jedoch auch, das Paradies wirklich zu verlassen, denn dieses ist, auch als ein anderes Paradies, ein denkbar ungeeigneter Ort, wie Lilian Peter jüngst in ihrem Essay Diebinnen im Paradies klargemacht hat: „Adam und Eva lebten im Garten Eden, bis Eva von einer Schlange überredet wurde, vom Baum der Erkenntnis eine Frucht zu stehlen und sich diese unwiederbringlich zu eigen $\mathrm{zu}$ machen. Gott, dessen uneingeschränktes Eigentum der Baum der Erkenntnis war, wurde daraufhin sehr böse, vertrieb Adam (hebräisch: der Mensch) und Eva (Bedeutung: nicht sicher) aus dem Paradies, was von Altgriechisch $\pi \alpha \rho \alpha \dot{\delta} \varepsilon\llcorner\sigma o \zeta=$ eingehegtes Gebiet, was von Hebräisch pardes, was von Altpersisch pairi-daeza = Ummauerung kommt, und verurteilte sie zum Tod. Seither gibt es das Gute und das Böse, Kluft zwischen Gott und Mensch, Mann und Frau, wobei das Böse die längste Zeit der Geschichte hindurch als Abfall vom Guten gedeutet wurde, d.h. als Diebstahl an der Einheit, wodurch die Differenz in die Welt kam, die anschließend immer als Differenz zwischen einem Über- und einer Unterlegenen (Gott und Mensch, Geist und Materie, Mann und Frau, Form und Stoff, Kultur und Natur usw.) gedacht wurde. ${ }^{10}$

Diese Differenz zwischen einem Über- und einer Unterlegenen hinter sich $\mathrm{zu}$ lassen, eröffnet eine weitere medienwissenschaftliche Perspektive und zwar die auf eine Teilhabe, mit der wihre medialen Bedingungen und Funktionslogik und damit die Möglichkeiten zur Unterscheidung zwischen Teilhabe und Nicht-Teilhabe, Inklusion und Exklusion durch die Beschreibung entsprechender Praktiken, Techniken und Objekten analysiert wird«, wie sie zum Beispiel Beate Ochsner, Isabell Otto und Markus Spöhrer vornehmen. ${ }^{11}$ Angewendet auf unsere Beispiele lässt sich so eine Logik der Ein- und Ausgrenzung hinterfragen (denken wir an Kieran, der davon erzählte, dass er ohne Prothese gehänselt

8 Tobias Nikolaus Klass, »Heterotopie«, in: Stephan Günzel (Hg.), Lexikon der Raumphilosophie, Darmstadt 2012, S. 173.

9 Vgl. Michel Foucault, Die Heterotopien. Der utopische Körper. Zwei Radiovorträge, Frankfurt/M. 2013.

10 Lilian Peter, Diebinnen im Paradies, Berlin 2018, S. 28-29.

11 Beate Ochsner, Isabell Otto, Markus Spöhrer, »Objekte medialer Teilhabe. Einleitung«, in: AugenBlick. Konstanzer Hefte zur Medienwissenschaft, Heft 58: Objekte medialer Teilhabe (2013), S. 6. 
wurde), in der Anormaliät von der Normalität aus festgestellt wird, was einer Monopolisierung der Normalität (oder auch der Vernunft) gleichkommt, gleichsam einem Logozentrismus statt eines dia-logos, der sich an Ansprüchen orientieren müsste beziehungsweise von diesen Ansprüchen überhaupt erst ins Werk gesetzt würde.

Teilhabe wird auf diese Weise zurückgeführt auf ihre antike griechische Anwendung als methexis: Wir partizipieren - meta - an der hexis - an der Haltung (von hexo, hexomai, shalten und nehmen eine Haltung ein, mit der wir in einer Spannung den anderen Menschen gegenüberstehen, uns in einer ontologischen Spannung befinden, wie sie Jean-Luc Nancy in Diskussion von Bild, Mimesis und Methexis vor allem in Bezug auf die Spannung zwischen Leben und Tod anhand der Totenmaske verdeutlicht, die uns am Tod teilhaben lässt. ${ }^{12}$ Auf der Seite der Lebenden bedeutet diese so über Verhaltungen und Anspannungen erfasste Teilhabe, sich an den konkreten Anderen in ihrer leiblichen Verfasstheit zu orientieren, im Zuge derer deutlich wird, dass Behinderung kein Objekt ist, das von außen den jeweiligen Menschen befällt, wie ein Virus einen biologischen Organismus, sondern eine ebenso vollständige Existenzform wie die einer Nicht-Behinderung ist, was etwa Martin Huth mit Maurice Merleau-Ponty deutlich macht: $\mathrm{Zu}$ unterscheiden ist mit Huth zwischen geistiger und körperlicher, erworbener und angeborener Behinderung, zwischen verschiedenen Formen der Beeinträchtigung und ihren individuellen Ausprägungen, die jedoch alle keine Verunmöglichung von Glück darstellen, auch wenn sie Einschränkungen in verschiedenen Zusammenhängen bedeuten, die von Technologien wie der Handprothese in Teilen kompensiert werden. ${ }^{13}$ Obwohl die Prothese vieles ermöglicht, wie das Fahrradfahren von Kieran und im Laufe ihrer Verwendung gar unauffällig oder transparent wird, irgendwie dazu gehört, so widersetzt sich ihre eigene Materialität auch immer wieder und macht sich bemerkbar, soweit sogar, dass sie als Eingriff in die eigene Integrität erlebt wird, nämlich in den Momenten, wo die auch brüchige Materialität die Kompensation unterminiert und Attribute wie snutzlos`, >ineffi-

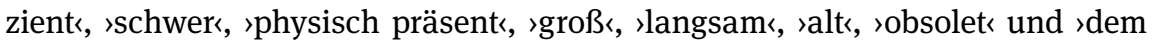
Zufall ausgesetzt in den Vordergrund rücken, was aber keinesfalls einen Mangel

12 Vgl. Jean-Luc Nancy, »Das Bild: Mimesis \& Methexis«, in: Emmanuel Alloa (Hg.), Bildtheorien aus Frankreich. Eine Anthologie, München 2011, S. 349-370.

13 Vgl. Martin Huth, "Phänomenologische Untersuchungen zum Thema Behinderung", in: Matthias Flatscher u.a. (Hg.), Neue Stimmen der Phänomenologie. Zweiter Band: Das Andere / Aisthesis, Nordhausen 2011, S. 342-355. Siehe außerdem Maurice Merleau-Ponty, Phänomenologie der Wahrnehmung, Berlin 1974. 
darstellt, denn immer wieder geht es mit der Prothese nicht nur um Kompensation oder auch Optimierung und Steigerung, wie sich zum Beispiel mit Karin Harrasser in Körper 2.0. Über die technische Erweiterbarkeit des Menschen weiter diskutieren ließe, ${ }^{14}$ sondern es geht ebenfalls darum, die Prothese willentlich abzulegen, ohne Kompensation auszukommen, keine Optimierung oder Steigerung anzustreben und situativ $\mathrm{zu}$ schauen, was $\mathrm{zu}$ tun oder nicht $\mathrm{zu}$ tun ist, aus eigener Aktivität und Passivität heraus den Dingen ihren freien Lauf zu lassen. ${ }^{15}$

Wir sind damit an einem Punkt angekommen, mit dem im zweiten und letzten Teil des Beitrags eine weitere Perspektive eingenommen wird, nämlich die Perspektive auf die Wirksamkeit, die ausgehend von Überlegungen von François Jullien skizziert werden soll.

\section{II.}

Mit Jullien zu arbeiten, bedeutet von Anfang an herauszustellen, dass es ihm um einen Ortswechsel des Denkens geht, den er mit einem Umweg über China vornimmt, im Rückgriff auf antike chinesische Philosophie, um die Ausgangsbedingungen der europäischen Vernunft zu hinterfragen, nicht jedoch, und das ist von großer Wichtigkeit, in einem Vergleich, sondern in einer Gegenüberstellung.

Ein zentrales Konzept Julliens ist in diesem Unterfangen die Wirksamkeit, mit der er unsere Augen auf das Modell richtet, auf die Modellbildung, die er in der europäischen Tradition, die zum Beispiel von Platons Ideenlehre (u.a. in Politeia/Der Staat, ca. 370 v. Chr.) überliefert wurde, als prägend ansieht: Wenn wir tätig werden, entwickeln wir eine Idealform (eidos), die wir als Ziel (telos) setzen, und dann handeln wir, um sie in die Realität umzusetzen. Unsere Augen sind im Handeln auf das Modell gerichtet. Die theoria als Schau der ewigen, unbewegten Ideen, geht der praxis voraus. Das Mittel folgt dem vorgefassten Zweck, der Entwurf bestimmt die Ausführung, das Projekt wird theoretisch

14 Vgl. Karin Harrasser, Körper 2.0. Über die technische Erweiterbarkeit des Menschen, Bielefeld 2013.

15 Ich denke hierbei an meine Erfahrungen in der >Alten`-, >Behinderten «- und `Kranken «-Pflege in Kiel und Berlin zwischen 1993 und 2007, vom Zivildienst bis nach der Promotion und als Lehrbeauftragter. Immer wieder geht es darum, aus dem eigenen und fremden leiblichen Selbst heraus zu existieren, es ohne Prothese zu probieren, sich auf sich einzulassen und sich auszuhalten, auch wenn der Gang in den Flur, das Trinken der Apfelschorle oder das Essen des Butterbrotes mißlingt, weil die Beinprothese, der `Schnabelbecher oder der Pfleger bewusst fehlen, nicht assistieren und keinesfalls optimieren sollen, obwohl damit ein Hinfallen, ein Verschütten oder ein Herunterfallen ermöglicht werden. 
entworfen, bevor wir es praktisch umsetzen. In Julliens Worten entsteht auf diese Weise Wirksamkeit dadurch, dass wir »eine vom Verstand entworfene und vom Willen als Ziel gesetzte Idealform versuchen, in die Wirklichkeit umzusetzen«. Im Denken antiker chinesischer Philosophie entsteht die Wirksamkeit demgegenüber »durch eine Logik der kontinuierlichen Veränderung der Dinge und der Situationen eher dadurch, dass wir früher oder später ausfindig machen, was in der Konfiguration tragend ist und uns dann von ihr tragen lassen, indem wir diese günstige Neigung ausnutzen. ${ }^{16}$

Es ist damit nicht die Projektion des Modells, wie das einer Handprothese, sondern die Neigung der Dinge, wie in der Situation des Jungen Kieran, die diesem Denken den Vorrang gibt, in der wir uns für eine bestimmte Richtung entscheiden. Jullien regt so zum Beispiel mit den Lehren des Laozi aus dem sechsten Jahrhundert vor Christi an, über die Effektivität des Realen nachzudenken, in der wir immer schon in bereits ablaufenden Prozessen stehen, im Zuge derer wir in jeder Situation ein Potenzial erkennen.

Doch wie erkennen wir ein solches Potenzial? Indem wir listig sind, und zwar nicht um Andere hereinzulegen, sondern um mit der Situation so umgehen zu können, dass wir auf die Logik ihres Ablaufs zählen und ihre Wirkung geschehen lassen, nicht auf sie drängen, sondern uns von ihr tragen lassen. Wir verstehen so die Wirksamkeit nicht nur techno-logisch und versuchen nicht, sie zu erzwingen, uns anzueignen oder auszuschöpfen. Vielmehr üben wir uns in Zurückhaltung.

In das Zentrum unserer Aufmerksamkeit rückt auf diese Weise nicht die Handlung oder die Erkenntnis, sondern der Weg, das tao. Dieser Ortswechsel im Denken Julliens, der einen anderen Weg einschlägt, birgt für eine medienwissenschaftliche Herangehensweise ein großes Potenzial, denn er versetzt ihre begrifflichen Ausgangsunterscheidungen inmitten von Theorie und Praxis und stellt sie auf einem fruchtbaren Spannungsfeld gegenüber: die Intentionalität mit der Performativität, die Teleologien mit dem Denken des Medialen, die Handlung mit Prozessen und Ereignissen, das Modell mit Basteln und Experimentieren. Mit Andreas Hetzel können wir an dieser Stelle von einer Transformation oder einer Schwerpunktverlagerung sprechen, die keinen Paradigmenwechsel nach sich zieht, sondern Beugungen und Brechungen erlaubt, vergleichbar eines Strahls, »der sich im Medium der Technik wie das weiße Licht in einem Prisma bricht. Statt der einen intendierten Wirkung stehen am Ende unserer heutigen, technisch vermittelten Handlungsketten vielfältig-bunte, schwer zu

16 François Jullien, Der Umweg über China. Ein Ortswechsel des Denkens, Berlin 2002, S. 48-49. 
kontrollierende Wirkungen, die nicht länger in Haupt- und Nebenfolgen aufgeteilt werden können. (17 $^{17}$

Wie oben schon angedeutet, geht es hier um die Gegenüberstellung auf diesem fruchtbaren Spannungsfeld, in der die Spannung gehalten werden soll, was nur aus der Gegenüberstellung heraus gelingt und nicht mit einer Unterscheidung, die in einem Vergleich, der in Beziehung setzt, zum Abschluss kommt, woraufhin jeder der beiden Pole seines Weges geht und sich in seiner Besonderheit einkapselt. Es ist daher der Abstand, den ich mit Jullien herstellen möchte, um die einmal unterschiedenen und getrennten Pole weiter im Blickfeld behalten zu können, denn nur durch ihn bleiben beide in der aufgetauchten Distanz in Gegenüberstellung zum anderen. Der Abstand ist daher keine Figur der Einordnung, mit der ich, um einen Vergleich anzustellen, einen hypothetischen gemeinsamen Rahmen setze, innerhalb dessen sich nach Gleichem und Anderem ordnen lässt, sondern der Abstand ist eine Figur der Störung und Unordnung. ${ }^{18}$

Bis zu welchem Punkt geht aber der Abstand? Bis zu welchem Punkt kann er gehen? Bis zu welchem Punkt lässt sich eine andere Möglichkeit der Zugänglichkeit, der Teilhabe und der Wirksamkeit, wie auch ihres Denkens entwickeln?

Der Abstand ist ein forschender Begriff, der eine heuristische Funktion hat, um in unangepasster Weise vorgehen zu können, Verschiebungen gegenüber Erwartetem und Vereinbartem vorzunehmen und sich an andere Orte vorzuwagen. Im Unterschied zur Differenz, die beide Pole auf die jeweilige Seite zurückfallen lässt, ist allein der Abstand in der Lage, wirklich ein Gemeinsames hervorzubringen. Im durch den Abstand eröffneten Zwischen können beide in eine Beziehung treten, ihre Selbstgenügsamkeit hinter sich lassen und die Begrenztheit ihres Selbstbezugs überwinden. Das durch den Abstand eröffnete Dazwischen bringt Jullien für unseren medienwissenschaftichen Kontext interessanterweise auf den griechischen Begriff metaxu, der bei Aristoteles in seiner Wahrnehmungslehre De Anima/Über die Seele (ca. in den 330er v. Chr.) auftaucht: ${ }^{19}$

Hinsichtlich des Sehens bedarf es eines Dritten, das dem Auge allererst erlaubt, etwas zu sehen. Dieses Dritte schiebt sich zwischen Auge und Gegen-

17 Andreas Hetzel, »Technik als Vermittlung und Dispositiv. Über die vielfältige Wirksamkeit der Maschinen«, in: Gerhard Gamm, Andreas Hetzel (Hg.), Unbestimmtheitssignaturen der Technik. Eine neue Deutung der technisierten Welt, Bielefeld 2005, S. 278.

18 Vgl. Jullien, Es gibt keine kulturelle Identität. Wir verteidigen die Ressourcen einer Kultur, Berlin 2017, S. 39 und ders., Der Weg zum Anderen. Alterität im Zeitalter der Globalisierung, Wien 2014, S. 34-35.

19 Vgl. Jullien, Der Weg zum Anderen, a.a.O., S. 53. 
stand und erlaubt die Vermittlung beider. Dahinter steckt die Einsicht, dass jedes Wahrgenommene sich dem Wahrnehmenden mitteilen muss, weswegen ein Drittes anzunehmen ist, wofür Aristoteles den Begriff metaxu verwendet, der aber unbestimmt bleibt. Er besitzt kein an und für sich, kein Sein oder eigenes Wesen und setzt sich als Mittleres oder Zwischenraum nur durch seine Gegensätze zusammen (im Mittelalter wird er daraufhin in den scholastischen Aristoteles-Kommentaren mit dem Begriff >Medium` belegt). »Nun«, fragt Jullien, was könnte dann aber eine »weitere Berufung auf dieses Dazwischen sein, wenn man es nicht mehr auf den Status eines Mittleren reduziert, zwischen plus und minus, sondern wenn es als durch zur Entfaltung kommt? « ${ }^{20}$ Da das Denken antiker chinesischer Philosophie sich in Begriffen von Hauch, Fluss und Atmung bewegt, ist es das Dazwischen, wovon und wodurch jede Begebenheit ausgeht und sich entwickelt. Die Wirkungsweise des Dazwischen ist so verstanden eine, die sich jeder konkreten Zuordnung entzieht; sie besteht vielmehr darin, Evasives, Ausflüchtendes zu erhalten, ohne dabei das Sinnliche zu verlassen oder auf die Phänomenalität der Dinge zu verzichten.

Haben wir damit einen weiteren, anderen Ort erreicht, wo Versuche der Teilhabe in technologischen Zeitaltern unternommen werden können? Wir können sagen ja, nur das dieses Dazwischen insofern kein anderer Ort sein kann, als dass es sich gar nicht an einem Ort befindet, sondern nirgendwo ist, in der, wie Jullien auch über Foucault hinausdenkt, A-topie, weswegen das Dazwischen niemals isolierbar ist, keine Eigenheiten besitzt, ohne Essenz und Beschaffenheit bleibt, aber dennoch funktioniert, kommuniziert und alles Treiben erst ermöglicht. Wir nehmen damit natürlich eine alles andere als standfeste Position ein, denn wir folgen so abwechselnd der Kohärenz der einen oder der anderen Denkweise, um sie in die Gegenüberstellung der Reflexion zu führen, die es uns, so gedacht, nicht erlaubt, uns auf irgendeiner Seite zu stabilisieren. Doch besteht darin nicht gerade die Aufgabe auch der Medienwissenschaft?

Angekommen sind wir hier bei der Erkenntnis, dass wir uns immer wieder damit begnügen müssen, dass wir, wie Dieter Mersch hervorhebt, »allenfalls Facetten, Aspekte oder Splitter dessen auffinden, was Medialität ausmacht, was auch heißt, im Unvollständigen zu verkehren und beständig neue Perspektiven aufzuschließen, eine besondere Aufmerksamkeit für das Nichtaufgehende und Widersprüchliche $\mathrm{zu}$ entwickeln und fortwährend andere Anfänge $\mathrm{zu}$ versuchen, im Metier der Schrift das Wissen um den beständigen Entzug wie auch die Heterogenität und theoretische Uneinholbarkeit nichtschriftlicher Medien wach

20 Ebd., S. 54. 
zu halten. ${ }^{21}$ Darin liegt die Funktion der Medienphilosophie, als Grundlegung der Epistemologie von Medienwissenschaft, als »Diskurs über ihre Begriffs- und Theoriebildungen, ihre Fundamente und Methoden wie deren Rechtfertigung. Insofern schließt Medienphilosophie an die klassischen Aufgaben von Philosophie an. ${ }^{22}$

Das Mediale wird immer wieder neu erkundet, wie gleichsam in einer psychoanalytischen Behandlung, in der es nicht darum geht, Eindeutigkeiten herauszuarbeiten, sondern zwischen Behandelter und Analytikerin ein Dazwischen einzuschalten. Das Mediale ist nicht das Eine, weswegen es auch nicht nur auf das Technologische reduziert werden kann, sondern im Denken einer Situation inmitten von Theorie und Praxis dazwischen wirkt, durch auf dem Weg genutztes oder auch ungenutztes Potenzial in Perspektivierungen auf Zugänglichkeit, Teilhabe und Wirksamkeit. Wir gewinnen so, über ein derart vorgeschlagenes Denken des Medialen, unsere Beschaffenheit aus dem Dazwischen des Unteruns.

Genau darüber aber ließe sich ıunterhalten`. Nehmen wir Jullien doch beim Wort. - Eine jede eröffnet der Anderen gegenüber ihre Position, entfaltet sie, legt sie frei und aktiviert sie durch sie. - Entre-tenir (unter-halten)? Dazwischen halten, im Dazwischen halten, ein Dazwischen in den Händen, in den Worten und Gesten halten...

\section{Literatur}

Coupland, Douglas, Mikrosklaven, München 1999.

Foucault, Michel, Die Heterotopien. Der utopische Körper. Zwei Radiovorträge, Frankfurt/M. 2013.

Harrasser, Karin, Körper 2.0. Über die technische Erweiterbarkeit des Menschen, Bielefeld 2013.

Hetzel, Andreas, »Technik als Vermittlung und Dispositiv. Über die vielfältige Wirksamkeit der Maschinen«, in: Gerhard Gamm, Andreas Hetzel (Hg.), Unbestimmtheitssignaturen der Technik. Eine neue Deutung der technisierten Welt, Bielefeld 2005, S. 275-296.

Huth, Martin, »Phänomenologische Untersuchungen zum Thema Behinderung«, in: Matthias Flatscher, Iris Laner, Martin Huth, Gerhard Thonhauser, Thomas Stadlbauer (Hg.), Neue

21 Dieter Mersch, „Wozu Medienphilosophie? Eine programmatische Einleitung«, in: ders., Michael Mayer (Hg.), Internationales Jahrbuch für Medienphilosophie, Band 1: Einschnitte: Zur Genesis und Geltung medienphilosophischer Reflexionen, Berlin/New York 2015, S. 46.

22 Ebd., S. 19. Jullien und Mersch treffen sich hier in der Gegenüberstellung mit Abstand im Denken des Dazwischen. 
Stimmen der Phänomenologie. Zweiter Band: Das Andere / Aisthesis, Nordhausen 2011, S. 342-355.

Jullien, François, Es gibt keine kulturelle Identität. Wir verteidigen die Ressourcen einer Kultur, Berlin 2017.

_Der Weg zum Anderen. Alterität im Zeitalter der Globalisierung, Wien 2014.

_Der Umweg über China. Ein Ortswechsel des Denkens, Berlin 2002.

—Über die Wirksamkeit, Berlin 1999.

Klass, Tobias Nikolaus, »Heterotopie«, in: Stephan Günzel (Hg.), Lexikon der Raumphilosophie, Darmstadt 2012, S. 172-173.

Merleau-Ponty, Maurice, Phänomenologie der Wahrnehmung, Berlin 1974.

Mersch, Dieter, „Wozu Medienphilosophie? Eine programmatische Einleitung «, in: Dieter Mersch, Michael Mayer (Hg.), Internationales Jahrbuch für Medienphilosophie, Band 1: Einschnitte: Zur Genesis und Geltung medienphilosophischer Reflexionen, Berlin/New York 2015, S. 13-48.

Nancy, Jean-Luc, »Das Bild: Mimesis \& Methexis«, in: Emmanuel Alloa (Hg.), Bildtheorien aus Frankreich. Eine Anthologie, München 2011, S. 349-370.

Ochsner, Beate, Isabell Otto, Markus Spöhrer, „Objekte medialer Teilhabe. Einleitung«, in: AugenBlick. Konstanzer Hefte zur Medienwissenschaft, Heft 58: Objekte medialer Teilhabe, Dezember 2013, S. 5-10.

Peter, Lilian, Diebinnen im Paradies, Berlin 2018.

Sequeira, Jessica, Other Paradises. Poetic Approaches to Thinking in a Technological Age, Winchester 2018. 
